\title{
PERIODIC SOLUTIONS OF SECOND-ORDER NONAUTONOMOUS DYNAMICAL SYSTEMS
}

\author{
MARTIN SCHECHTER
}

Received 13 March 2006; Revised 10 May 2006; Accepted 15 May 2006

We study the existence of periodic solutions for second-order nonautonomous dynamical systems. We give four sets of hypotheses which guarantee the existence of solutions. We were able to weaken the hypotheses considerably from those used previously for such systems. We employ a new saddle point theorem using linking methods.

Copyright (c) 2006 Martin Schechter. This is an open access article distributed under the Creative Commons Attribution License, which permits unrestricted use, distribution, and reproduction in any medium, provided the original work is properly cited.

\section{Introduction}

We consider the following problem. One wishes to solve

$$
-x^{\prime \prime}(t)=\nabla_{x} V(t, x(t))
$$

where

$$
x(t)=\left(x_{1}(t), \ldots, x_{n}(t)\right)
$$

is a map from $I=[0, T]$ to $\mathbb{R}^{n}$ such that each component $x_{j}(t)$ is a periodic function in $H^{1}$ with period $T$, and the function $V(t, x)=V\left(t, x_{1}, \ldots, x_{n}\right)$ is continuous from $\mathbb{R}^{n+1}$ to $\mathbb{R}$ with

$$
\nabla_{x} V(t, x)=\left(\frac{\partial V}{\partial x_{1}}, \ldots, \frac{\partial V}{\partial x_{n}}\right) \in C\left(\mathbb{R}^{n+1}, \mathbb{R}^{n}\right)
$$

Here $H^{1}$ represents the Hilbert space of periodic functions in $L^{2}(I)$ with generalized derivatives in $L^{2}(I)$. The scalar product is given by

$$
(u, v)_{H^{1}}=\left(u^{\prime}, v^{\prime}\right)+(u, v) .
$$

For each $x \in \mathbb{R}^{n}$, the function $V(t, x)$ is periodic in $t$ with period $T$. 
2 Periodic solutions of second-order nonautonomous dynamical systems

We will study this problem under the following assumptions:

(1)

$$
V(t, x) \geq 0, \quad t \in I, x \in \mathbb{R}^{n}
$$

(2) there are constants $m>0, \alpha \leq 6 m^{2} / T^{2}$ such that

$$
V(t, x) \leq \alpha, \quad|x| \leq m, t \in I, x \in \mathbb{R}^{n}
$$

(3) there is a constant $\mu>2$ such that

$$
\begin{gathered}
\frac{H_{\mu}(t, x)}{|x|^{2}} \leq W(t) \in L^{1}(I), \quad|x| \geq C, t \in I, x \in \mathbb{R}^{n}, \\
\limsup _{|x| \rightarrow \infty} \frac{H_{\mu}(t, x)}{|x|^{2}} \leq 0,
\end{gathered}
$$

where

$$
H_{\mu}(t, x)=\mu V(t, x)-\nabla_{x} V(t, x) \cdot x
$$

(4) there is a subset $e \subset I$ of positive measure such that

$$
\liminf _{|x| \rightarrow \infty} \frac{V(t, x)}{|x|^{2}}>0, \quad t \in e .
$$

We have the following theorem.

Theorem 1.1. Under the above hypotheses, the system (1.1) has a solution.

As a variant of Theorem 1.1, we have the following one.

Theorem 1.2. The conclusion in Theorem 1.1 is the same if Hypothesis (2) is replaced by

$\left(2^{\prime}\right)$ there is a constant $q>2$ such that

$$
V(t, x) \leq C\left(|x|^{q}+1\right), \quad t \in I, x \in \mathbb{R}^{n},
$$

and there are constants $m>0, \alpha<2 \pi^{2} / T^{2}$ such that

$$
V(t, x) \leq \alpha|x|^{2}, \quad|x| \leq m, t \in I, x \in \mathbb{R}^{n} .
$$

We also have the following theorem.

Theorem 1.3. The conclusions of Theorems 1.1 and 1.2 hold if Hypothesis (3) is replaced by

$\left(3^{\prime}\right)$ there is a constant $\mu<2$ such that

$$
\begin{gathered}
\frac{H_{\mu}(t, x)}{|x|^{2}} \geq-W(t) \in L^{1}(I), \quad|x| \geq C, t \in I, x \in \mathbb{R}^{n}, \\
\liminf _{|x| \rightarrow \infty} \frac{H_{\mu}(t, x)}{|x|^{2}} \geq 0 .
\end{gathered}
$$


And we have the following theorem.

THeOREM 1.4. The conclusion of Theorem 1.1 holds if Hypothesis (1) is replaced by $\left(1^{\prime}\right)$

$$
0 \leq V(t, x) \leq C\left(|x|^{2}+1\right), \quad t \in I, x \in \mathbb{R}^{n}
$$

and Hypothesis (3) by

$\left(3^{\prime \prime}\right)$ the function given by

$$
H(t, x)=2 V(t, x)-\nabla_{x} V(t, x) \cdot x
$$

satisfies

$$
\begin{gathered}
H(t, x) \leq W(t) \in L^{1}(I), \quad|x| \geq C, t \in I, x \in \mathbb{R}^{n}, \\
H(t, x) \longrightarrow-\infty, \quad|x| \longrightarrow \infty, t \in I, x \in \mathbb{R}^{n} .
\end{gathered}
$$

The periodic nonautonomous problem

$$
x^{\prime \prime}(t)=\nabla_{x} V(t, x(t))
$$

has an extensive history in the case of singular systems (cf., e.g., Ambrosetti-Coti Zelati [1]). The first to consider it for potentials satisfying (1.3) were Berger and Schechter [3]. We proved the existence of solutions to (1.17) under the condition that

$$
V(t, x) \longrightarrow \infty \text { as }|x| \longrightarrow \infty
$$

uniformly for a.e. $t \in I$. Subsequently, Willem [16], Mawhin [6], Mawhin and Willem [8], Tang [11, 12], Tang and $\mathrm{Wu}[13-15], \mathrm{Wu}$ and Tang [17] and others proved existence under various conditions (cf. the references given in these publications).

The periodic problem (1.1) was studied by Mawhin and Willem [7, 8], Long [5], Tang and $\mathrm{Wu}$ [13-15] and others (cf. the refernces quoted in them). Ben-Naoum et al. [2] and Nirenberg (cf. Ekeland and Ghoussoub [4]) proved the existence of nonconstant solutions.

We will prove Theorems 1.1-1.4 in the next section. We use a linking method of critical point theory (cf. $[9,10])$. These methods allow us to improve the previous results.

\section{Proofs of the theorems}

We now give the proof of Theorem 1.1.

Proof. Let $X$ be the set of vector functions $x(t)$ given by (1.2) and described above. It is a Hilbert space with norm satisfying

$$
\|x\|_{X}^{2}=\sum_{j=1}^{n}\left\|x_{j}\right\|_{H^{1}}^{2} .
$$


4 Periodic solutions of second-order nonautonomous dynamical systems

We also write

$$
\|x\|^{2}=\sum_{j=1}^{n}\left\|x_{j}\right\|^{2}
$$

where $\|\cdot\|$ is the $L^{2}(I)$ norm.

Let

$$
N=\left\{x(t) \in X: x_{j}(t) \equiv \text { constant, } 1 \leq j \leq n\right\}
$$

and $M=N^{\perp}$. The dimension of $N$ is $n$, and $X=M \oplus N$. Proof of the following lemma can be found in [7].

LEMmA 2.1. If $x \in M$, then

$$
\|x\|_{\infty}^{2} \leq \frac{T}{12}\left\|x^{\prime}\right\|^{2}, \quad\|x\| \leq \frac{T}{2 \pi}\left\|x^{\prime}\right\|
$$

We define

$$
G(x)=\left\|x^{\prime}\right\|^{2}-2 \int_{I} V(t, x(t)) d t, \quad x \in X
$$

For each $x \in X$ write $x=v+w$, where $v \in N, w \in M$. For convenience, we will use the following equivalent norm for $X$ :

$$
\|x\|_{X}^{2}=\left\|w^{\prime}\right\|^{2}+\|v\|^{2} .
$$

If $x \in M$ and

$$
\left\|x^{\prime}\right\|^{2}=\rho^{2}=\frac{12}{T} m^{2}
$$

then Lemma 2.1 implies that $\|x\|_{\infty} \leq m$, and we have by Hypothesis (2) that $V(t, x) \leq \alpha$. Hence,

$$
G(x) \geq\left\|x^{\prime}\right\|^{2}-2 \int_{|x(t)|<m} \alpha d t \geq \rho^{2}-2 \alpha T \geq 0 .
$$

We also note that Hypothesis (1) implies

$$
G(v) \leq 0, \quad v \in N .
$$

Take

$$
A=\partial B_{\rho} \cap M, \quad \rho^{2}=\frac{12}{T} m^{2}, \quad B=N,
$$

where

$$
B_{\sigma}=\left\{x \in X:\|x\|_{X}<\sigma\right\} .
$$


By [9, Theorem 1.1], $A$ links $B$. (For background material on linking theory, cf. [10].) Moreover, by (2.8) and (2.9), we have

$$
\sup _{A}[-G] \leq 0 \leq \inf _{B}[-G]
$$

Hence, we may apply [9, Theorem 1.1] to conclude that there is a sequence $\left\{x^{(k)}\right\} \subset X$ such that

$$
\begin{gathered}
G\left(x^{(k)}\right)=\left\|\left[x^{(k)}\right]^{\prime}\right\|^{2}-2 \int_{I} V\left(t, x^{(k)}(t)\right) d t \rightarrow c \leq 0, \\
\frac{\left(G^{\prime}\left(x^{(k)}\right), z\right)}{2}=\left(\left[x^{(k)}\right]^{\prime}, z^{\prime}\right)-\int_{I} \nabla_{x} V\left(t, x^{(k)}(t)\right) \cdot z(t) d t \rightarrow 0, \quad z \in X, \\
\frac{\left(G^{\prime}\left(x^{(k)}\right), x^{(k)}\right)}{2}=\left\|\left[x^{(k)}\right]^{\prime}\right\|^{2}-\int_{I} \nabla_{x} V\left(t, x^{(k)}(t)\right) \cdot x^{(k)}(t) d t \longrightarrow 0 .
\end{gathered}
$$

If

$$
\rho_{k}=\left\|x^{(k)}\right\|_{X} \leq C
$$

then there is a renamed subsequence such that $x^{(k)}$ converges to a limit $x \in X$ weakly in $X$ and uniformly on $I$. From (2.14) we see that

$$
\frac{\left(G^{\prime}(x), z\right)}{2}=\left(x^{\prime}, z^{\prime}\right)-\int_{I} \nabla_{x} V(t, x(t)) \cdot z(t) d t=0, \quad z \in X,
$$

from which we conclude easily that $x$ is a solution of (1.1).

If

$$
\rho_{k}=\left\|x^{(k)}\right\|_{X} \longrightarrow \infty
$$

let $\tilde{x}^{(k)}=x^{(k)} / \rho_{k}$. Then, $\left\|\tilde{x}^{(k)}\right\|_{X}=1$. Let $\tilde{x}^{(k)}=\widetilde{w}^{(k)}+\widetilde{v}^{(k)}$, where $\tilde{w}^{(k)} \in M$ and $\tilde{v}^{(k)} \in N$. There is a renamed subsequence such that $\left\|\left[\tilde{x}^{(k)}\right]^{\prime}\right\| \rightarrow r$ and $\left\|\tilde{x}^{(k)}\right\| \rightarrow \tau$, where $r^{2}+\tau^{2}=1$. From (2.13) and (2.15) we obtain

$$
\begin{gathered}
\left\|\left[\tilde{x}^{(k)}\right]^{\prime}\right\|^{2}-\frac{2 \int_{I} V\left(t, x^{(k)}(t)\right) d t}{\rho_{k}^{2}} \longrightarrow 0, \\
\left\|\left[\tilde{x}^{(k)}\right]^{\prime}\right\|^{2}-\frac{\int_{I} \nabla_{x} V\left(t, x^{(k)}(t)\right) \cdot x^{(k)}(t) d t}{\rho_{k}^{2}} \longrightarrow 0 .
\end{gathered}
$$

Thus,

$$
\begin{gathered}
\frac{2 \int_{I} V\left(t, x^{(k)}(t)\right) d t}{\rho_{k}^{2}} \longrightarrow r^{2}, \\
\frac{\int_{I} \nabla_{x} V\left(t, x^{(k)}(t)\right) \cdot x^{(k)}(t) d t}{\rho_{k}^{2}} \longrightarrow r^{2} .
\end{gathered}
$$


6 Periodic solutions of second-order nonautonomous dynamical systems

Hence, by (1.9),

$$
\frac{\int_{I} H_{\mu}\left(t, x^{(k)}(t)\right) d t}{\rho_{k}^{2}} \longrightarrow\left(\frac{\mu}{2}-1\right) r^{2}
$$

Note that

$$
\left|\tilde{x}^{(k)}(t)\right| \leq C|| \tilde{x}^{(k)} \|_{X}=C .
$$

If

$$
\left|x^{(k)}(t)\right| \longrightarrow \infty
$$

then by (1.8)

$$
\limsup \frac{H_{\mu}\left(t, x^{(k)}(t)\right)}{\rho_{k}^{2}}=\limsup \frac{H_{\mu}\left(t, x^{(k)}(t)\right)}{\left|x^{(k)}(t)\right|^{2}}\left|\tilde{x}^{(k)}(t)\right|^{2} \leq 0 .
$$

If

$$
\left|x^{(k)}(t)\right| \leq C
$$

then

$$
\frac{H_{\mu}\left(t, x^{(k)}(t)\right)}{\rho_{k}^{2}} \longrightarrow 0
$$

Hence,

$$
\limsup \frac{\int_{I} H_{\mu}\left(t, x^{(k)}(t)\right) d t}{\rho_{k}^{2}} \leq 0
$$

Hence by (2.22)

$$
\left(\frac{\mu}{2}-1\right) r^{2} \leq 0
$$

If $r \neq 0$, this contradicts the fact that $\mu>2$. If $r=0$, then $\widetilde{w}^{(k)} \rightarrow 0$ uniformly in $I$ by Lemma 2.1. Moreover, $T\left|\widetilde{v}^{(k)}\right|^{2}=\left\|\widetilde{v}^{(k)}\right\|^{2} \rightarrow 1$. Hence, there is a renamed subsequence such that $\tilde{v}^{(k)} \rightarrow \tilde{v}$ in $N$ with $|\tilde{v}|^{2}=1 / T$. Hence, $\tilde{x}^{(k)} \rightarrow \tilde{v}$ uniformly in $I$. Consequently, $\left|x^{(k)}\right| \rightarrow \infty$ uniformly in $I$. Thus, by Hypothesis (4),

$$
\liminf \frac{\int_{I} V\left(t, x^{(k)}(t)\right) d t}{\rho_{k}^{2}} \geq \int_{e} \liminf \frac{V\left(t, x^{(k)}(t)\right)}{\left|x^{(k)}(t)\right|^{2}}\left|\tilde{x}^{(k)}(t)\right|^{2} d t>0
$$

This contradicts (2.20). Hence the $\rho_{k}$ are bounded, and the proof is complete. 
The proof of Theorem 1.2 is similar to that of Theorem 1.1 with the exception of the inequality (2.8) resulting from Hypothesis (2). In its place we reason as follows: if $x \in M$, we have by Hypothesis $\left(2^{\prime}\right)$,

$$
\begin{aligned}
G(x) & \geq\left\|x^{\prime}\right\|^{2}-2 \int_{|x|<m} \alpha|x(t)|^{2} d t-2 C \int_{|x(t)|>m}\left(|x(t)|^{q}+1\right) d t \\
& \geq\left\|x^{\prime}\right\|^{2}-2 \alpha\|x\|^{2}-2 C\left(1+m^{-q}\right) \int_{|x(t)|>m}|x(t)|^{q} d t \\
& \geq\left\|x^{\prime}\right\|^{2}\left(1-\left[\frac{2 \alpha T^{2}}{4 \pi^{2}}\right]\right)-C^{\prime} \int_{|x(t)|>m}|x(t)|^{q} d t \\
& \geq\left(1-\left[\frac{\alpha T^{2}}{2 \pi^{2}}\right]\right)\|x\|_{X}^{2}-C^{\prime \prime} \int_{I}\|x\|_{X}^{q} d t \\
& \geq\left(1-\left[\frac{\alpha T^{2}}{2 \pi^{2}}\right]\right)\|x\|_{X}^{2}-C^{\prime \prime \prime}\|x\|_{X}^{q} \\
& =\left(1-\left[\frac{\alpha T^{2}}{2 \pi^{2}}\right]-C^{\prime \prime \prime}\|x\|_{X}^{q-2}\right)\|x\|_{X}^{2}
\end{aligned}
$$

by Lemma 2.1. Hence, we have the following lemma.

LEMMA 2.2.

$$
G(x) \geq \varepsilon\|x\|_{X}^{2}, \quad\|x\|_{X} \leq \rho, x \in M
$$

for $\rho>0$ sufficiently small, where $\varepsilon<1-\left[\alpha T^{2} / 2 \pi^{2}\right]$.

The remainder of the proof is essentially the same.

In proving Theorem 1.3 we follow the proof of Theorem 1.1 until we reach (2.20). Then we reason as follows. If

$$
\left|x^{(k)}(t)\right| \longrightarrow \infty
$$

then

$$
\liminf \frac{H_{\mu}\left(t, x^{(k)}(t)\right)}{\rho_{k}^{2}}=\liminf \frac{H_{\mu}\left(t, x^{(k)}(t)\right)}{\left|x^{(k)}(t)\right|^{2}}\left|\tilde{x}^{(k)}(t)\right|^{2} \geq 0 .
$$

If

$$
\left|x^{(k)}(t)\right| \leq C,
$$

then by Hypothesis $\left(3^{\prime}\right)$,

$$
\frac{H_{\mu}\left(t, x^{(k)}(t)\right)}{\rho_{k}^{2}} \longrightarrow 0
$$

Hence,

$$
\liminf \frac{\int_{I} H_{\mu}\left(t, x^{(k)}(t)\right) d t}{\rho_{k}^{2}} \geq 0
$$


8 Periodic solutions of second-order nonautonomous dynamical systems

Thus by (2.22)

$$
\left(\frac{\mu}{2}-1\right) r^{2} \geq 0
$$

If $r \neq 0$, this contradicts the fact that $\mu<2$. If $r=0$, then $\widetilde{w}^{(k)} \rightarrow 0$ uniformly in $I$ by Lemma 2.1. Moreover, $T\left|\widetilde{v}^{(k)}\right|^{2}=\left\|\widetilde{v}^{(k)}\right\|^{2} \rightarrow 1$. Hence, there is a renamed subsequence such that $\tilde{v}^{(k)} \rightarrow \tilde{v}$ in $N$ with $|\widetilde{v}|^{2}=1 / T$. Hence, $\tilde{x}^{(k)} \rightarrow \widetilde{v}$ uniformly in $I$. Consequently, $\left|x^{(k)}\right| \rightarrow \infty$ uniformly in $I$. Thus, by Hypothesis (4),

$$
\liminf \frac{\int_{I} V\left(t, x^{(k)}(t)\right) d t}{\rho_{k}^{2}} \geq \int_{e} \liminf \frac{V\left(t, x^{(k)}(t)\right)}{\left|x^{(k)}(t)\right|^{2}}\left|\tilde{x}^{(k)}(t)\right|^{2} d t>0
$$

This contradicts (2.20). Hence the $\rho_{k}$ are bounded, and the proof is complete.

In proving Theorem 1.4, we follow the proof of Theorem 1.1 until (2.20). Assume first that $r>0$. Note that (2.13) and (2.15) imply that

$$
\int_{I} H\left(t, x^{(k)}(t)\right) d t \longrightarrow-c
$$

On the other hand, by Hypothesis $\left(1^{\prime}\right)$, we have

$$
\begin{aligned}
0 & \longleftarrow\left\|\left[\tilde{x}^{(k)}\right]^{\prime}\right\|^{2}-2 \int_{I} \frac{V\left(t, x^{(k)}(t)\right) d t}{\rho_{k}^{2}} \\
& \geq\left\|\left[\tilde{x}^{(k)}\right]^{\prime}\right\|^{2}-2 C \int_{I}\left(\left|\tilde{x}^{(k)}(t)\right|^{2}+\rho_{k}^{-2}\right) d t \\
& \longrightarrow r^{2}-2 C \int_{I}|\tilde{x}(t)|^{2} d t .
\end{aligned}
$$

Hence, $\tilde{x}(t) \neq \equiv$. Let $\Omega_{0} \subset I$ be the set on which $\tilde{x}(t) \neq 0$. The measure of $\Omega_{0}$ is positive. Moreover, $\left|x^{(k)}(t)\right| \rightarrow \infty$ as $k \rightarrow \infty$ for $t \in \Omega_{0}$. Thus,

$$
\int_{I} H\left(t, x^{(k)}(t)\right) d t \leq \int_{\Omega_{0}} H\left(t, x^{(k)}(t)\right) d t+\int_{I \backslash \Omega_{0}} W(t) d t \longrightarrow-\infty
$$

by Hypothesis $\left(3^{\prime \prime}\right)$. But this contradicts (2.40). If $r=0$, then $\widetilde{w}^{(k)} \rightarrow 0$ uniformly in $I$ by Lemma 2.1. Moreover, $T\left|\widetilde{v}^{(k)}\right|^{2}=\left\|\tilde{v}^{(k)}\right\|^{2} \rightarrow 1$. Thus, there is a renamed subsequence such that $\widetilde{v}^{(k)} \rightarrow \widetilde{v}$ in $N$ with $|\widetilde{v}|^{2}=1 / T$. Hence, $\tilde{x}^{(k)}(t) \rightarrow \widetilde{v}$ uniformly in $I$. Consequently, $\left|x^{(k)}(t)\right| \rightarrow \infty$ uniformly in $I$. Thus, by Hypothesis (4),

$$
\liminf \frac{\int_{I} V\left(t, x^{(k)}(t)\right) d t}{\rho_{k}^{2}} \geq \int_{e} \liminf \frac{V\left(t, x^{(k)}(t)\right)}{\left|x^{(k)}(t)\right|^{2}}\left|\tilde{x}^{(k)}(t)\right|^{2} d t>0
$$

This contradicts (2.20). Hence the $\rho_{k}$ are bounded, and the proof is complete. 


\section{References}

[1] A. Ambrosetti and V. Coti Zelati, Periodic Solutions of Singular Lagrangian Systems, Progress in Nonlinear Differential Equations and Their Applications, vol. 10, Birkhäuser Boston, Massachusetts, 1993.

[2] A. K. Ben-Naoum, C. Troestler, and M. Willem, Existence and multiplicity results for homogeneous second order differential equations, Journal of Differential Equations 112 (1994), no. 1, 239-249.

[3] M. S. Berger and M. Schechter, On the solvability of semilinear gradient operator equations, Advances in Mathematics 25 (1977), no. 2, 97-132.

[4] I. Ekeland and N. Ghoussoub, Selected new aspects of the calculus of variations in the large, Bulletin of the American Mathematical Society 39 (2002), no. 2, 207-265.

[5] Y. M. Long, Nonlinear oscillations for classical Hamiltonian systems with bi-even subquadratic potentials, Nonlinear Analysis 24 (1995), no. 12, 1665-1671.

[6] J. Mawhin, Semicoercive monotone variational problems, Académie Royale de Belgique. Bulletin de la Classe des Sciences 73 (1987), no. 3-4, 118-130.

[7] J. Mawhin and M. Willem, Critical points of convex perturbations of some indefinite quadratic forms and semilinear boundary value problems at resonance, Annales de l'Institut Henri Poincaré. Analyse Non Linéaire 3 (1986), no. 6, 431-453.

[8] Critical Point Theory and Hamiltonian Systems, Applied Mathematical Sciences, vol. 74, Springer, New York, 1989.

[9] M. Schechter, New linking theorems, Rendiconti del Seminario Matematico della Università di Padova 99 (1998), 255-269.

[10]_, Linking Methods in Critical Point Theory, Birkhäuser Boston, Massachusetts, 1999.

[11] C.-L. Tang, Periodic solutions of non-autonomous second order systems with $\gamma$-quasisubadditive potential, Journal of Mathematical Analysis and Applications 189 (1995), no. 3, 671-675.

[12] _ Periodic solutions for nonautonomous second order systems with sublinear nonlinearity, Proceedings of the American Mathematical Society 126 (1998), no. 11, 3263-3270.

[13] C.-L. Tang and X.-P. Wu, Periodic solutions for second order systems with not uniformly coercive potential, Journal of Mathematical Analysis and Applications 259 (2001), no. 2, 386-397.

[14]_, Periodic solutions for a class of nonautonomous subquadratic second order Hamiltonian systems, Journal of Mathematical Analysis and Applications 275 (2002), no. 2, 870-882.

[15] N__ Notes on periodic solutions of subquadratic second order systems, Journal of Mathematical Analysis and Applications 285 (2003), no. 1, 8-16.

[16] W. Willem, Oscillations forcées systèmes hamiltoniens, Public. Sémin. Analyse Non Linéarie, Université de Franche-Comté, Besancon, 1981.

[17] X.-P. Wu and C.-L. Tang, Periodic solutions of a class of non-autonomous second-order systems, Journal of Mathematical Analysis and Applications 236 (1999), no. 2, 227-235.

Martin Schechter: Department of Mathematics, University of California, Irvine,

CA 92697-3875, USA

E-mail address: mschecht@math.uci.edu 